\title{
Fuzzy Expert System for Severity Measurement of COVID-19 Suspect
}

\author{
B.T. Jadhav, G.S. Nhivekar \\ Department of Electronics, Yashavantrao Chavan Institute of Science, Satara, India \\ Corresponding Author: G.S. Nhivekar
}

\begin{abstract}
The pandemic of COVID-19 disease is spread over the world. The symptoms of COVID-19 disease can vary from mild to severe illness. Also, these symptoms are complex and uncertain in nature. The severity score is useful to treat the suspect that highly depends on symptoms. To handle with this problem, the current study makes use of the Fuzzy Expert System which is one in every of the foremost suitable methods in modelling systems with high uncertainty and complexity. In this study, the fuzzy-based expert system is designed to measure the severity of COVID-19 disease in suspect.
\end{abstract}

Keywords: Fuzzy Logic, Expert System, COVID-19

\section{INTRODUCTION}

In Wuhan city, Hubei province and reported to the WHO Country Office in China on 31 December 2019 that the novel COVID-19 (severe acute respiratory syndrome coronavirus-2) disease was detected. Since then it's spread to Globe [1].

The virus COVID-19 may be a RNA (ribonucleic acid) virus. It's a member of the Coronavirus family and frequently it's found in mammals and birds. Within the past, spilling of the members this virus over between species that's mammals and birds to humans [1] Many individuals infected with COVID-19 experienced mainly respiratory symptoms fever, dry cough, Headache, severe breathing difficulties and fatigue; a number of them are required Oxygen moreover as mechanical ventilation [2, 3].
Infection of Covid-19 varies from Mild to critical. Mild to critical is that the severity scale of COVID-19 disease. Report on the severity of the Covid-19 disease given by the Chinese Center for Disease Control and Prevention with features Mild (80.9\%), severe disease (13.8\%), and demanding disease $(4.7 \%)$ with a death rate of $2.3 \%$ [1].

Manually Prediction of severity of covid -19 patients could be a challenging problem. Hence, it's an important and challenging problem to recognize the patients with high risk of death from the infectious population using testing data with the help of a fuzzy expert system. The developed fuzzy experts system could initiate early treatment to patients and thus potentially minimize the mortality.

The PCR test directly detects the presence of the virus RNA. Hence, it's a preliminary test for COVID-19. An efficient method of early detection of infection is that the Lung CT is additionally proposed by Ali et al $[6,7]$.

Patients must choose clinical testing after test confirmation of infection, to spot the chance factors for severe illness. After the study analysis of Covid-19 patients, serious illness is found in patients of all ages, but it arises mostly in patients with underlying medical disorders and with old age patients. That the previous reports of clinical characteristics, medical complexities and medical reports are often a good technique to predict the severity of illness of covid-19 patients [1] 
By employing a set of clinical parameters, a fuzzy algorithm can identify the amount of various parameters of the patient which contribute to identification of severity of covid -19 patients within the variety of mild to critical. Fuzzy algorithms give the score to the applied parameters with their contribution in critical illness of the infected patient.

To reduce the morbidity, early assessing of the severity of covid-19 infected patients for the classification of patient death risk is extremely important which can help to sort and treated patients to urge perfect clinical management with use of available medical facilities and forestall the under handling of patient care. To conduct an assessment of treatment and severity is important for optimizing the allocation of rescue resources and to prevent the looks of under treatment patients, where within the medical emergency occurs and medical resources don't seem to be fulfilling the service to the patients. within the field of health look after detection, identification, and estimation of varied medical problems for the clinical characterization no techniques have gain popularity like computing (AI) techniques like Support Vector Machines (SVM), Decision Trees, formal logic, Deep learning have gained popularity, Genetic algorithms (GA), and Artificial Neural Networks (ANNs) $[1,3,4,5]$.

This paper discusses the study that has a primary objective to use and evaluate a fuzzy logic technique for precise prediction of the severity of the COVID-19 disease in suspects. The clinical findings were accustomed train different supervised mathematical logic techniques, and some of knowledge sets were accustomed evaluate the effectiveness of the proposed methods.

\section{MATERIALS \& METHODS}

The fuzzy expert system used during this work has three blocks which include Fuzzification, knowledge inference, and Defuzzification. Figure. 1 shows the methods used for the study.

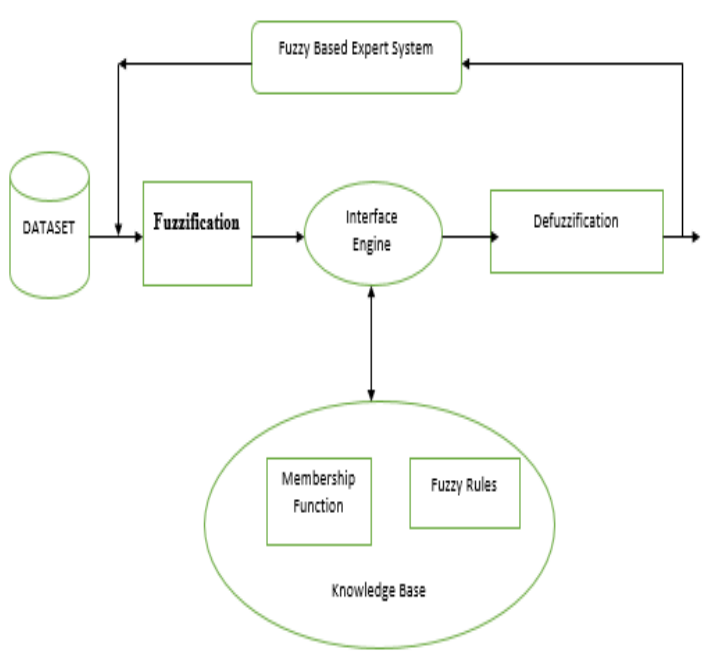

Figure1: the methods used for the study

a) Fuzzification: Fuzzification is the method of converting a crisp input value to a fuzzy value that's performed by the utilization of the data within the cognitive content. Converting a Crisp set to Fuzzy Set is additionally referred to as Fuzzification. In case of digital logic which has either 0 or 1 value ie true or false, Fuzzy logic has infinite numbers from 0 to 1 . Fuzzy sets allow membership function value that may be in between 0 and 1. Fuzzy logic is simply an expression of vagueness and uncertainty. The matter of accurate boundaries and overlapping will be avoided using this method. Therefore, the system's inputs and output were fuzzified so as to handle the inaccuracies, ambiguities, and uncertainties related to severity higher cognitive process of Covid-19 patients [9].

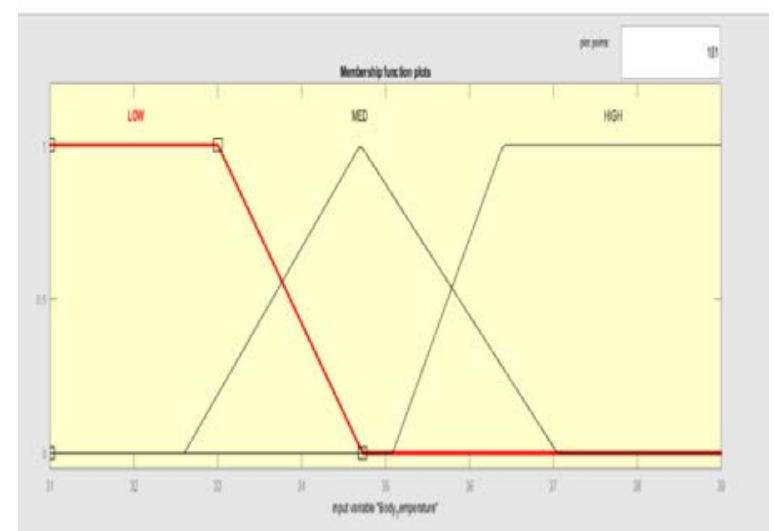

Figure2: Membership functions of the linguistic variables Temperature 


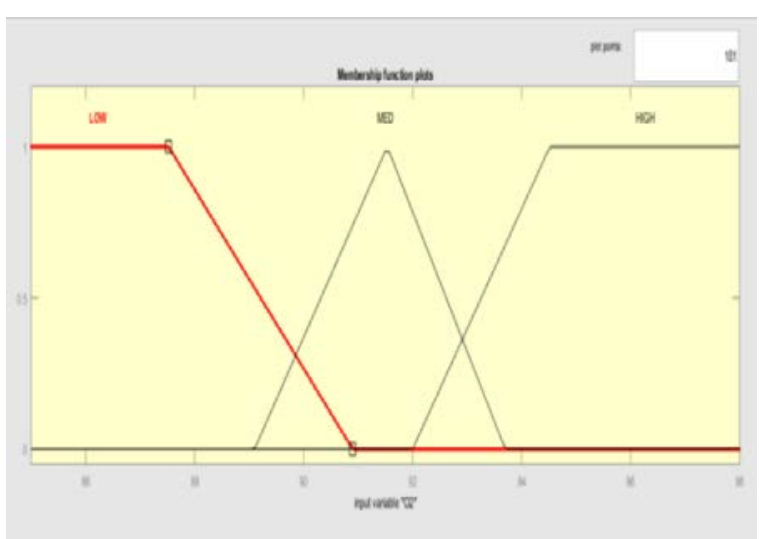

Figure 3: Membership functions of the linguistic variables Oxygen Level

In the present system input parameters include: temperature, oxygen level and hart rate which are defined with three fuzzy linguistic values. The output variable severity with an input parameter has three fuzzy values. The output variable severity diagnosis has normal, medium, and high fuzzy linguistic values. By the fuzzy membership grade, the value of a fuzzy variable is defined. For all input variables a trapezoidal and triangular membership function was used, while the triangular membership function was used for the output variable. A mathematical expression for trapezoidal membership function is represented as Trapezoidal (x; a,b,c,d). Each crisp value has been converted into a fuzzy value. Table 1 shows the fuzzy rules, Figure. 2 shows membership functions and linguistic variables Temperature, Figure. 3 shows the membership function of the linguistic variables Oxygen Level and Figure. 4 shows the membership functions of the linguistic variables vital sign. Figure. 5 shows the membership functions of the output linguistic variables Severity.

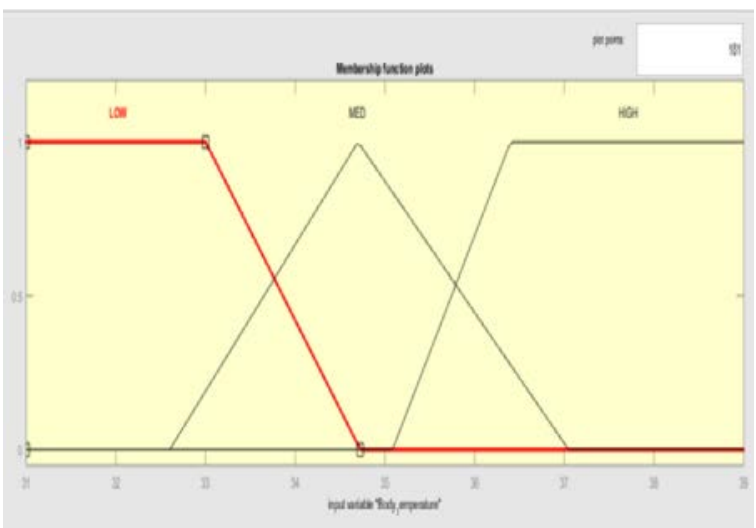

Figure 4: Membership functions of the linguistic variables Pulse Rate

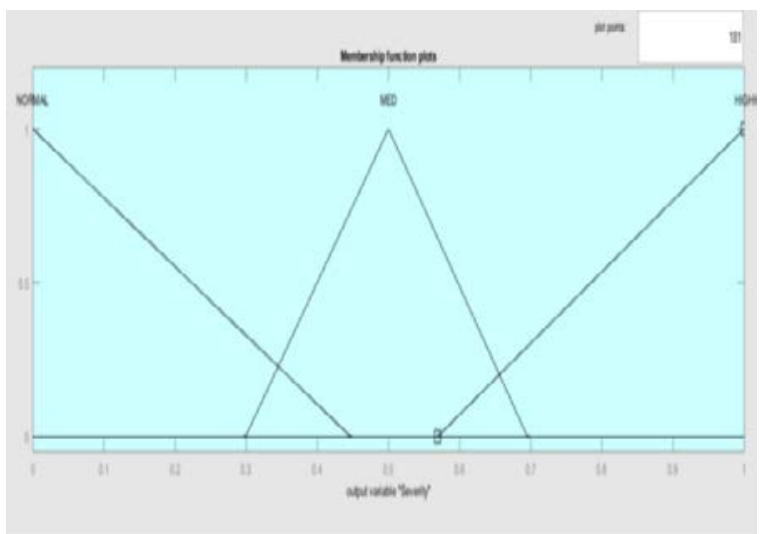

Figure 5: Membership functions of the output linguistic variables Severity

\begin{tabular}{|c|c|c|c|c|}
\hline \multirow{2}{*}{$\begin{array}{l}\text { Rule } \\
\text { Number }\end{array}$} & \multicolumn{3}{|c|}{ Linguistic Inputs } & \multirow{2}{*}{$\begin{array}{c}\text { Linguistic Output } \\
\text { Severity } \\
\end{array}$} \\
\hline & Temperature & Oxygen Level & Pulse Rate & \\
\hline 1 & LOW & LOW & LOW & MED \\
\hline 2 & LOW & LOW & MED & MED \\
\hline 3 & LOW & LOW & HIGH & HIGH \\
\hline 4 & LOW & MED & LOW & MED \\
\hline 5 & LOW & MED & MED & MED \\
\hline 6 & LOW & MED & HIGH & MED \\
\hline 7 & LOW & HIGH & LOW & NORMAL \\
\hline 8 & LOW & HIGH & MED & NORMAL \\
\hline 9 & LOW & HIGH & HIGH & MED \\
\hline 10 & MED & LOW & LOW & MED \\
\hline 11 & MED & LOW & MED & MED \\
\hline 12 & MED & LOW & HIGH & HIGH \\
\hline 13 & MED & MED & LOW & NORMAL \\
\hline 14 & MED & MED & MED & MED \\
\hline 15 & MED & MED & HIGH & MED \\
\hline 16 & MED & HIGH & LOW & NORMAL \\
\hline 17 & MED & HIIGH & MED & NORMAL \\
\hline 18 & MED & HIGH & HIGH & MED \\
\hline 19 & HIGH & LOW & LOW & HIGH \\
\hline
\end{tabular}




\begin{tabular}{|l|l|l|l|l|}
\hline \multicolumn{5}{|c|}{ Table no.1 continued.... } \\
\hline 20 & HIGH & LOW & MED & HIGH \\
\hline 21 & HIGH & LOW & HIGH & HIGH \\
\hline 22 & HIGH & MED & LOW & MED \\
\hline 23 & HIGH & MED & MED & MED \\
\hline 24 & HIGH & MED & HIGH & HIGH \\
\hline 25 & HIGH & HIGH & LOW & MED \\
\hline 26 & HIGH & HIGH & MED & MED \\
\hline 27 & HIGH & HIGH & HIGH & MED \\
\hline
\end{tabular}

b) Knowledge inference: In fuzzy logic systems new knowledge is generated from existing fuzzy rules available in the system knowledge base. Therefore, new information and conclusions would be realized from it. In this work Mamdani inference technique is used to stimulate reasoning in identification of severity of Covid-19 patients. Due to good results with simple structure, the Mamdani Fuzzy Inference method is widely used. In the present system Mamdani technique is used [9]. Figure. 6 shows the Graphical User Interface (GUI) of System using Mamdani technique.

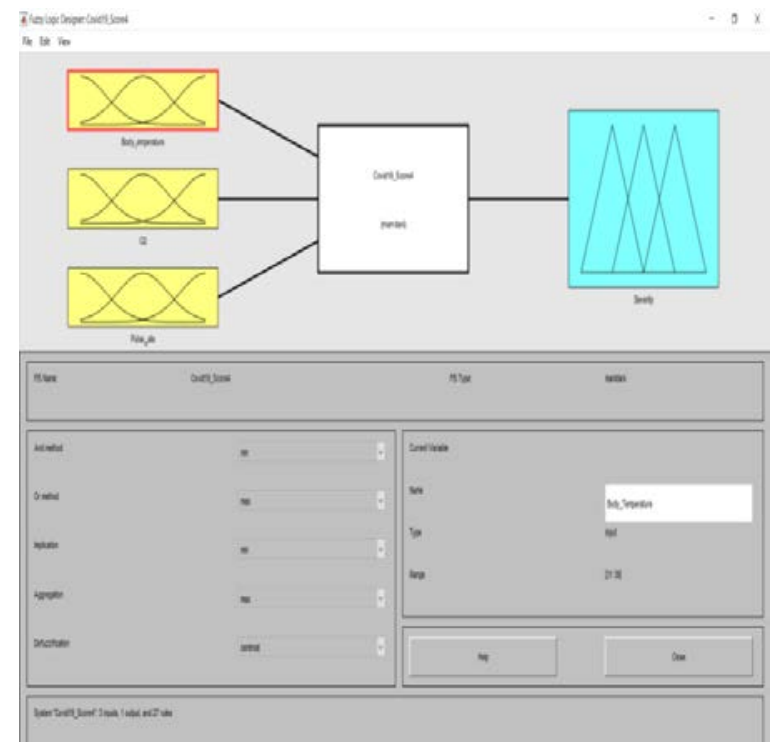

Figure6: Graphical User Interface (GUI) of the System Inference with Mamdani Technique.

C) Defuzzification: Defuzzification of clipped fuzzy sets generated in the fuzzy reasoning process yields the single crisp value of severity condition that represents the aggregate fuzzy outcome. The Centroid defuzzification method being simple yet stands as a good compromise of all other methods. Defuzzification involved the process that transforms fuzzy values into crisp values. For the Defuzzification process the present system uses the Centroid method. The Centroid method determines the center-of-area of fuzzy set and gives the corresponding crisp value which is the output of the system. Fuzzy expert system is designed to identify severity of Covid-19 and simulated by using the fuzzy rule viewer as shown in Figure. 7. The Rule Viewer displays a roadmap of the whole fuzzy inference process. The Rule Viewer allows the analysis of the complete fuzzy inference process. The Rule Viewer also shows how the overall result is affected due to the shape of membership functions. It consists of 27 rules with three inputs and one output. The inputs consist of symptoms in Table 1, body temperature, Oxygen level (O2) and Pulse rate while the output is the risk of COVID-19 (\%). Linguistic variables; HIGH, MEDIUM, LOW are used for the output which can be interpreted as, Infected, Likely Infected, and Nearly Absent.

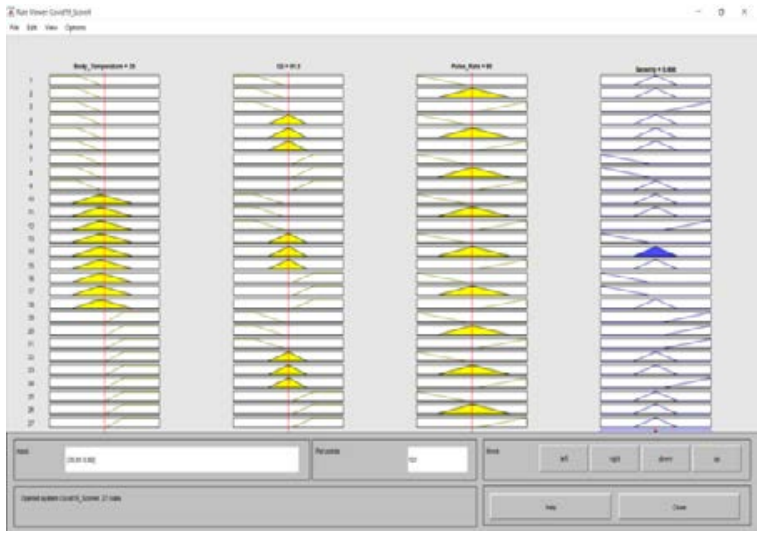

Figure7: Graphical User Interface (GUI) of Rule Viewer

Figure. 8 shows the 3 -dimensional View of the system. The full range of the output set is based on the full range of the input set. The surface viewer depicts the dependencies of output (covide-19 severity Condition) on the input that is body Temperature, O2 level and pulse rate as illustrated in Figure. 8. This 3-dimensional 
plot signifies the mapping from body Temperature, $\mathrm{O} 2$ level and pulse rate to the individuals 'severity Condition.

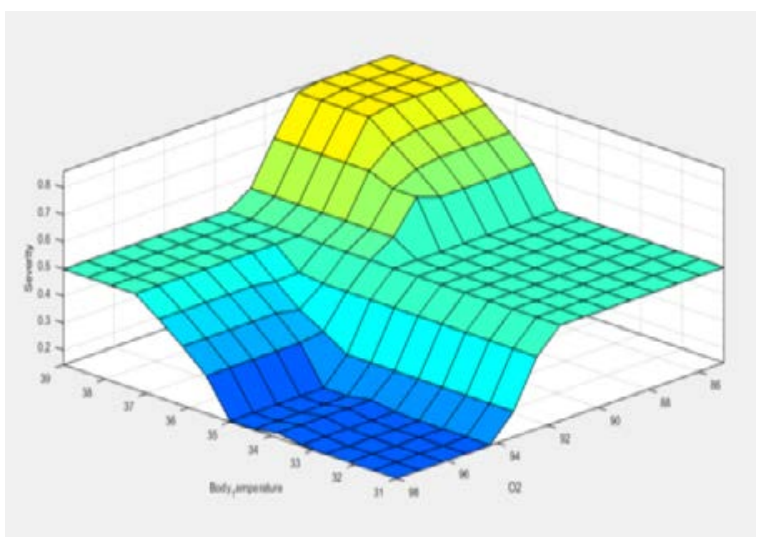

Figure 8: GUI of 3-dimensional plot

Fuzzy Expert System Simulink model used for the thorough testing of the system performance by providing the different types of inputs. The model also includes the setup for comparing the variation in performance output after different alteration or tuning in multiple FIS. The model is designed to view the resultant output of the FIS while provided with the input data. For input clinical parameter random number generator is used the resulting output of FES severity is shown in Figure.9.

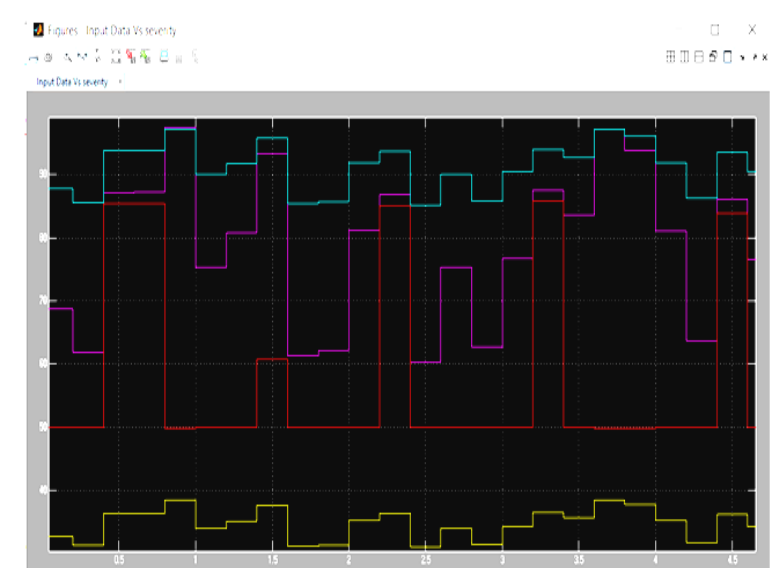

Figure9: Fuzzy Expert System severity output for different input data

\section{CONCLUSION}

The Fuzzy Expert System (FES) allows obtaining a prediction of Covide-19 disease risk without health professionals. The input data to FES can be obtained by the readily available instrument that doesn't need an expert to handle. The developed Fuzzy Expert system is useful to provide early evaluation of individuals' COVID-19 health status. For future work, the system will be integrated with the Internet of Things that make it available remotely for the user

Acknowledgement: None

\section{Conflict of Interest: None}

\section{Source of Funding: None}

\section{REFERENCES}

1. Aziz Alotaibi, Mohammad Shiblee, Adel Alshahrani 'Prediction of Severity of COVID-19-Infected Patients Using Machine Learning Techniques' Computers 2021, $10, \quad 31$. https://doi.org/10.3390/computers10030031

2. Mohammad Asaduzzaman Chowdhury, Quazi Zubair Shah Mohammod Abul Kashem,Abdus Shahid, and Nasim Akhtar2"Evaluation of the Effect of Environmental Parameters on the Spread of COVID-19: A Fuzzy Logic Approach, Advances in Fuzzy Systems"Volume 2020,Article ID 8829227, 5 pages https://doi.org/10.1155/2020/8829227

3. Abd-alrazaq, Alaa \& Alajlani, Mohannad \& Alhuwail, Dari \& Schneider, Jens \& AlKuwari, Saif \& Shah, Zubair \& Hamdi, Mounir \& Househ, Mowafa. (2020). Artificial Intelligence in the Figureht against COVID-19: A Scoping Review (Preprint). Journal of Medical Internet Research. vol.22,No.12, 2021.DOI:10.2196/20756

4. Ali Narin, Ceren Kaya, Ziynet Pamuk "Automatic Detection of Coronavirus Disease (COVID-19) Using X-ray Images and Deep Convolutional Neural Networks" Pattern Analysis and Applications, vol.24, No.3, pp.1207-1220, 2021.DOI. 10.1007/s 10044-021-00984-y

5. M. Saqib Nawaz1, Philippe Fournier-Viger "Using artificial intelligence techniques for COVID-19” Applied Intelligence, vol. 51, pp.3086-3103, 2021.

6. Mannudeep K. Kalra \& Fatemeh Homayounieh \& Chiara Arru1\& Ola Holmberg \& Jenia Vassileva "Chest CT practice and protocols for COVID-19 from radiation dose management perspective" 
European Radiology, vol. 30,pp.6554-6560. 2020.

7. Ali Asadollahi-Amin, Mehrdad Hasibi, Fatemeh Ghadimi "Lung Involvement Found on Chest CT Scan in a PreSymptomatic Person with SARS-CoV-2 Infection: A Case Report Trop.” Med. Infect.Dis.,vol.5,No.562020, DOI:10.3390/tropicalmed5020056.

8. Pius Uagbae Ejodamen, Victor Eshiet Ekong “A Fuzzy Expert System Model for the Determination of Coronavirus Disease Risk” International Journal of Mechatronics, Electrical and Computer Technology (IJMEC), vol. 11, No.39, pp. 4825-4831, 2021.

9. Muhammad, L.J., Algehyne, E.A. Fuzzy based expert system for diagnosis of coronary artery disease in nigeria. Health Technol. Vol.11, pp.319-329, 2021. DOI: 10.1007/s12553-021-00531-z

10. Allam, Fayrouz, Zaki Nossair, Hesham Gomma, Ibrahim Ibrahim, and Mona Abdelsalam. "Evaluation of using a recurrent neural network (RNN) and a fuzzy logic controller (FLC) in closed loop system to regulate blood glucose for type-1 diabetic patients." International Journal of Intelligent Systems and Applications vol 4, No. 10, pp 58-71, 2012. DOI: 10.5815/ijisa.2012.10.07

11. Mamoria, Pushpa, and Deepa Raj. "Comparison of mamdani fuzzy inference system for multiple membership functions." International Journal of Image, Graphics and Signal Processing vol 8, No. 92016 pp 26-30. DOI: 10.5815/ijigsp.2016.09.04

12. Anurag Tatkare, Hemangi Patil, Tejal Salunke, Shreya Warang, Dipak Marathe "COVID-19 Patient Health Monitoring
System" vol 8, No. 9 ,pp 48-55. 2016. DOI: 10.5815/ijigsp.2016.09.04

13. Mirza Waseem Hussain, Tabasum Mirza, Malik Mubasher Hassan. "Impact of COVID-19 Pandemic on the Human Behavior", International Journal of Education and Management Engineering (IJEME), Vol.10, No.5, pp.35-61, 2020. DOI: 10.5815/ijeme.2020.05.05

14. : Kakelli Anil Kumar, Omkar Dhadge,"A Novel Infrared (IR) Based Sensor System for Human Presence Detection in Targeted Locations", International Journal of Computer Network and Information Security(IJCNIS), Vol.10, No.12, pp.34-40, 2018.DOI: 10.5815/ijcnis.2018.12.04

15. Ahmed Hassan Mohammed Hassan, Arfan Ali Mohammed Qasem, Walaa Faisal Mohammed Abdalla, Omer H. Elhassan, "Visualization \& Prediction of COVID-19 Future Outbreak by Using Machine Learning", International Journal of Information Technology and Computer Science(IJITCS), Vol.13, No.3, pp.16-32, 2021. DOI: $10.5815 /$ ijitcs.2021.03.02

16. İbrahim Berkan Aydilek, Abdülkadir Gümüşçü, "Designing a Rule Based Expert Systems for Contact Lenses Patients", Technology and Computer Science (IJITCS), Vol.10, No.3,pp.18-26, 2018. DOI: 10.5815/ ijitcs.2018.03.03

How to cite this article: B.T. Jadhav, G.S. Nhivekar. Fuzzy expert system for severity measurement of Covid-19 suspect. International Journal of Research and Review. 2021; 8(12): 139-144. DOI: https://doi.org/10.52403/ijrr. 20211219 